\title{
LA EDUCACIÓN INTERCULTURAL Y LAS IDENTIDADES DE GÉNERO, CLASE Y ETNIA
}

\author{
Horacio Gómez Lara \\ golahora33@yahoo.com.mx \\ CESMECA \\ Universidad de Ciencias y Artes de Chiapas \\ México
}

\section{RESUMEN}

Este trabajo hace referencia a la oportunidad que puede representar la educación intercultural para la resignificación de las identidades de género, etnia y clase. Se reflexiona a partir de la investigación sobre educación realizada en el municipio de San Andrés Larráinzar o Sakamch'en de los Pobres, Chiapas. A partir de los resultados de investigación se argumenta que en el contexto actual en que se está practicando la educación intercultural bilingüe en Chiapas y en el marco de políticas educativas que no son construcciones propias de los pueblos indígenas significan nada más nuevas imposiciones simuladas con los discursos de respeto a la diversidad cultural.

Palabras clave: educación, interculturalidad, identidades, pueblos indígenas. 


\section{SUMMARY}

This paper refers to the opportunity that can represent the intercultural education for remeaning the gender, class and ethnic identities. The work is a reflection from the investigation made in the municipality of San Andrés Larráinzar or Sakamch'en in Chiapas. From the results of investigation is argued that in the present context that the intercultural bilingual education is practicing in Chiapas and in the context of educational policies are not people's own constructions, mean nothing more new impositions simulated with the speeches of respect for cultural diversity.

Key words: education, intercultural, identities, indigenous people. 


\section{INTRODUCCIÓN}

Este artículo forma parte de un trabajo que había quedado en el tintero desde ya hace algunos años. Aprovecho la invitación de los coordinadores de este número de la Revista Pueblos y fronteras digital para que se difunda como parte de lo que considero un proceso de recibir-devolver a las y los habitantes de San Andrés Sakamch'en de los Pobres, o San Andrés Larráinzar, ${ }^{1}$ en el estado de Chiapas, el conocimiento que adquirí en este pueblo durante una etapa de trabajo de campo, entre el año 2000 y 2001. El artículo integra las reflexiones que en aquel entonces me suscitó el proyecto recién formulado de educación intercultural bilingüe, como proyecto educativo que se comenzaba a plantear para nuestro país y que, según lo percibo, sigue teniendo la misma vigencia.

Al igual que entonces, sigo considerando que la construcción de un nuevo proyecto educativo es una tarea indispensable, porque de otra forma no se podrá avanzar en la construcción del respeto efectivo a las diversas culturas que conformando la nación mexicana la enriquecen con las identidades indígenas y mestizas; mismas que constituyen, en su conjunto, la pregonada identidad nacional. Pero como parte de esa misma diversidad se requiere, en el caso de las culturas indígenas y mestizas pobres que, en el aspecto educativo, económico, social, cultural, se les otorgue atención especial, no paternalista, por los rezagos que en la calidad de vida han acumulado y que como clase y etnia no les ha permitido participar de manera equitativa y en un plano de igualdad con el resto de la población. Asimismo, al interior de cada cultura se requiere de una revisión de las relaciones de género que desfavorecen a las mujeres y conceden el domino a los hombres.

\section{LA EDUCACIÓN INTERCULTURAL BILINGÜE}

Como punto de partida y con la intención de aportar elementos que contribuyan a la reflexión sobre la práctica de la educación intercultural bilingüe para las diversas culturas indígenas de nuestro país y de la educación intercultural para el resto de la población mexicana, ${ }^{2}$ considero necesario retomar algunas cuestiones acerca de los conceptos de multiculturalidad, pluriculturalidad e interculturalidad que en los últimos años han venido circulando en el ámbito de la educación. En una ponencia que presentó Sylvia Schmelkes, ${ }^{3}$ 
en julio de 2001, hacía alusión precisamente a las diferencias entre los conceptos referidos, en este sentido señalaba que:

\begin{abstract}
De alguna manera, la multiculturalidad o la pluriculturalidad supone la coexistencia de diversas culturas en un determinado territorio, como tal, este concepto de multiculturalidad no implica el respeto que debe tener una cultura respecto de la otra; yo creo que tenemos que aceptar que en las realidades multiculturales, cuando solamente se definen así, existen profundas asimetrías, es decir, relaciones de poder, entre las culturas que discriminan a unas culturas en relación con otras, y entonces se generan relaciones de segregación y de discriminación cuando simplemente nos contentamos con una realidad multicultural, yo creo que es lo que nosotros podemos ver cuando analizamos lo que ocurre con las culturas indígenas en México y en América Latina en general (Schmelkes 2001: 1).
\end{abstract}

Acorde con la opinión que, desde sus inicios, se ha venido generalizando en el ámbito educativo, el concepto de interculturalidad trata, en sí mismo, de trascender el concepto de multiculturalidad o pluriculturalidad. Y lo trata de trascender en tanto que reconoce que los integrantes de las culturas, en la multiculturalidad, se relacionan en planos de desigualdad. El multiculturalismo lleva a límites extremos las diferencias, culturalizando las desigualdades sociales, de género y clase, fragmentando de ese modo la vida social.

La interculturalidad, como modelo utópico, supone que las personas de las distintas culturas se relacionarán en planos de igualdad, sin relaciones de poder de por medio, y que la diversidad misma es una riqueza y su reconocimiento una necesidad para que las culturas transiten hacia la democracia (Schmelkes 2001: 2).

Pero, ¿cuál es el contexto actual en que se enmarca el proyecto educativo intercultural que se plantea para nuestro país?, ¿es la educación intercultural bilingüie un nuevo integracionismo neoliberal? El proyecto intercultural está enmarcado en los procesos de globalización y de neoliberalismo que actúan como paradigmas impuestos por la modernidad. Desde el marco de la globalización, los programas políticos del Estado siguen siendo imposiciones; para ejemplificar esto, tomamos como referencia el controvertido Plan Puebla Panamá, PPP, ${ }^{4}$ con cuyos objetivos pretende, a través de la educación, atacar 
los problemas socioeconómicos que aquejan a la población del sur-sureste mexicano, concibiendo a la educación como capacitación para el trabajo, en áreas específicas:

La educación se considera instrumento fundamental para el desarrollo humano individual y social. La región Sur-Sureste llegará tan lejos como llegue su educación. Dado que los problemas y el rezago educativo que presenta la región son aún más graves que los de otras áreas del país, se requiere un programa articulado que permita atacarlos de manera especial. El índice de analfabetismo del Sur-Sureste es diez puntos porcentuales mayor que el promedio del resto de México. Su índice de escolaridad media es de sólo 6,7 años, mientras que la del resto del país es de 8.1 años (1er. Objetivo básico del PPP).

Tal parece que la educación, en estas regiones, no llegará muy lejos, pues ya a finales de la década de 1970 se había empleado este mismo lema —el de «llegar tan lejos como llegue la educación»- en el Programa para la Atención a las Zonas Marginadas del país.

Un Estado como el mexicano, que se considera democrático, no debería estar en permanente contradicción, señalando en sus discursos la equidad, la igualdad social de las, los gobernados, la práctica de consensos, de responder a las demandas sociales, mientras por otra parte siguen imponiendo las políticas de Estado excluyentes y discriminatorias que han caracterizado nuestro devenir histórico. El Plan Puebla Panamá, como todos los planes neoliberales, responde a las necesidades del orden mundial actual, a la nueva división internacional del trabajo, que se empeña en regionalizar las necesidades del mercado mundial, capacitando y especializando a sectores de la Población Económicamente Activa, PEA, en determinadas competencias laborales — por ejemplo, la formación de recursos humanos técnicos - utilizando, para tal fin, la educación como instrumento de capacitación. En estas políticas se concibe la educación como un producto más del mercado, como ha alertado la relatora de la Comisión de Derechos Humanos de ONU sobre el derecho a la educación, Katarina Tomasevski. ${ }^{5}$ 
INTERCULTURALIDAD EN UN CONTEXTO DE DESIGUALDAD: EL CASO DE SAN ANDRÉS SAKAMCH'EN DE LOS POBRES O SAN ANDRÉS LARRÁINZAR, CHIAPAS

Desarrollo este apartado refiriéndome a la educación en el municipio de San Andrés Larráinzar, como un resumen de lo que constituyó mi tesis de licenciatura, que fue donde surgieron estas reflexiones. En dicha tesis (Gómez 2002) analicé tres subespacios o subcampos, según la teoría y metodología de Pierre Bourdieu, que en su conjunto constituyen el campo de la educación en el municipio, estos son: la educación tradicional indígena, ETI; la educación escolar, y la educación autónoma.

\section{EDUCACIÓN TRADICIONAL INDÍGENA, ETI}

La educación tradicional indígena es el proceso que ha permitido hasta la actualidad la existencia de lo que conocemos como culturas indígenas, es decir, el proceso dialéctico en el que han reproducido sus particulares formas de vida, cosmovisión del mundo, valores culturales, tradiciones y costumbres. Para esto las familias y las comunidades indígenas —instituciones principales de ETI - han jugado el papel de maestras colectivas del proceso de enseñanza-aprendizaje de las nuevas generaciones; sin embargo, las nuevas generaciones también han participado de manera activa en el proceso de reproducción cultural al resignificar, rechazar o asumir como dado por naturaleza — prescripción trascendente - las enseñanzas del deber ser y el hacer de los hombres y mujeres en la tierra. Por lo anterior, ETI puede considerarse como el proceso mediante el cual las generaciones adultas enseñan a los niños y niñas el modelo socialmente aceptado de ser indígena y miembro de la comunidad, para llegar a ser bats 'i viniketik, hombres verdaderos, y antsetik, mujeres verdaderas, y alcanzar la virtud de los totil-meil, los padres-madres, ancestros.

De esta forma, las y los agentes que participan en ETI centran sus esfuerzos en reproducir el grupo familiar, y con ello la comunidad, a través de la enseñanza de sus particulares formas de vida, cosmovisión del mundo, rituales y prácticas cotidianas, como el lenguaje, el trabajo, la convivencia en los espacios físicos inmediatos: la casa, la comunidad y el municipio. Los rituales de conformación de una nueva familia, por ejemplo el matrimonio, el nacimiento de los primogénitos, juegan un papel fundamental en el proceso de ETI; por 
ejemplo, el ritual del nacimiento de un/a niño/a es un momento importante para la pareja, existen un sin número de creencias que acompañan todo el proceso de embarazo. Sin embargo, una práctica que resulta trascendental es el momento mismo del parto, puesto que a partir de ese momento se comienzan a construir las identidades de género - teniendo como justificación «natural» de esta diferenciación el sexo del (la) recién nacido/a «...y, muy especialmente, la diferencia "anatómica" entre los órganos sexuales puede aparecer de ese modo como la justificación natural de la diferencia socialmente establecida entre los sexos, y en especial de la división sexual del trabajo» (Bourdieu 2000: 24)—, con la asignación simbólica de las herramientas que corresponden al niño — machete, azadón, coa, etc. - o a la niña —utensilios de cocina, huso para hilar, etc.—. Como mandato trascendente se imponen los papeles sociales que han de asumir los niños y las niñas y que los familiares adultos se encargarán — de igual forma como mandato trascendente- de inculcar y vigilar que se cumplan.

El niño o la niña — junto a su madre - tiene sus primeras experiencias educativas; a partir de que aprenden a movilizarse por sí mismos comienza el proceso de socialización que es un segundo nivel de las relaciones que los vincula a la comunidad y donde continúa el aprendizaje de las reglas, prescripciones y formas de comportamiento socialmente aceptadas, que contribuyen a integrarlo y adquirir las gramáticas comunicacionales de la vida en comunidad. La pedagogía local existe como un proceso que, como práctica trascendente, permite la reproducción social y cultural de las y los indígenas, este se basa en cuatro principios, que metodológicamente permiten la formación de los bats'i viniketik y las antsetik, cuyo objetivo final es la búsqueda del modelo ideal intersubjetivo que busca el equilibrio a partir de las relaciones entre tres elementos: naturaleza-personas-deidades; estos deben relacionarse de manera correlativa para lograr el equilibrio cósmico. Los principios pedagógicos ${ }^{6}$ de ETI son, a saber:

\section{EL CONCEPTO DE RECIPROCIDAD: DAR Y RECIBIR CONSEJOS}

La concepción indígena de reciprocidad señala que, en el aspecto de la formación de los hombres y mujeres para la correcta vida en familia y en comunidad, los consejos, las enseñanzas que un día recibieron los que ahora fungen como maestros/as de los/as jóvenes 
y niños, es decir, los padres, principalmente, aunque también participan los/as abuelos/as y tíos/as, deben fomentar - enseñar-devolver - las conductas socialmente aceptadas por las familias y la comunidad.

\section{LA FORMACIÓN DE LA CONCIENCIA, CH'ULEL}

El concepto de alma, ch'ulel, entre los grupos indígenas tsotsiles, se entiende como una entidad sobrenatural con tres significaciones: la primera entendida como la vida misma, kushlejal, la fuerza que dinamiza y hace existir, el alma que tienen todos los seres de la tierra, humanos, vegetales, animales e inertes, como las piedras (Pérez 2000: 96-97); la segunda significación se refiere a la existencia de un animal compañero, vayjel, protector.

La preservación de la identidad del vayjel equivale a la protección de la vida misma de su portador/a. En San Andrés se cree que hay seres humanos que pueden tener hasta trece animales compañeros, vayjeletik. La morada de los vayjeletik son las montañas sagradas, tetik, desde donde vigilan los comportamientos humanos; el tercer significado del ch'ulel se refiere a la conciencia o responsabilidad de los seres humanos. Este significado se relaciona más directamente con la pedagogía local y con la moral — relacionado directamente con el cuarto principio- que se pretende inculcar a los miembros de la cultura. Pues el moldeamiento del ch'ulel es el que hará a una persona responsable de sus actos, que acatará las normatividades impuestas y aceptadas por todos los miembros de la comunidad, sólo así será una persona a la que le ha llegado su sch’ulel, su alma: conciencia (Pérez 2000: 97).

\section{LA CAPACITACIÓN FÍSICA Y MENTAL}

Como proceso integral, en ETI, los niños y niñas desarrollan sus potencialidades físicas y mentales a través de actividades prácticas donde pueden construir sus cuerpos. Dos actividades fundamentales para esto son los juegos y el trabajo. Existe una separación genérica de los juegos, por lo regular los niños prefieren jugar «juegos de niños», y las niñas «juegos de niñas», como actividades naturalizadas en lo social (Gómez 2002). Mediante la práctica recreativa los niños y niñas van formando sus identidades genéricas, dado que los juegos, en la mayoría de los casos, son representaciones infantiles de las actividades laborales que observan de su padre y madre. Las actividades laborales que, 
paulatinamente, los padres les van asignando, de acuerdo con su sexo y edad, contribuyen también a la construcción de sus cuerpos. A partir de que los padres consideran que los niños o niñas deben comenzar a aprender su «oficio», para los niños el trabajo en el campo y para las niñas el apoyo a su mamá en los trabajos de la casa; es entonces cuando el juego pasa a un segundo plano con el propósito de que los niños y niñas adquieran la responsabilidad del trabajo.

\section{LA FORMACIÓN DE LA CONDUCTA: ENDEREZAR EL CORAZÓN}

Existe otro proceso formativo en ETI. Este se refiere al proceso de inculcación-introyección de los valores considerados como positivos en los espacios sociales indígenas, que son, a saber: respeto, estima, ayuda mutua y honradez. La persona que posee un «corazón recto» es capaz de comprender el sufrimiento de los demás y se ofrece para ayudar a corregir los errores y problemas de sus semejantes, además muestran gran temperancia y tolerancia, no se molestan con facilidad ante actitudes arbitrarias de sus semejantes. El proceso de enderezar el corazón es para toda la vida, porque los seres humanos trabajamos, pensamos, erramos y nos corregimos mediante los consejos permanentes de los que nos rodean (Pérez 2000: 104). Una persona que se molesta con facilidad, que no acepta los consejos de sus padres o de las personas con quienes convive es alguien que «no sirve su corazón», es de corazón pequeño.

Todos los principios pedagógicos de ETI están interrelacionados y de manera paralela contribuyen a la formación de los hombres y las mujeres que la familia y la comunidad aspiran a formar.

\section{EDUCACIÓN ESCOLAR OFICIAL}

En la cabecera del municipio se encuentran en funcionamiento dos escuelas primarias del subsistema federal - monolingües, con enseñanza exclusiva en castellano y con maestros mestizos que llegan, la mayor parte, de San Cristóbal de Las Casas-. La cabecera municipal también cuenta con una secundaria técnica que se fundó a principios de la década de 1980; y ahora cuenta con el nivel de bachillerato, a través de una institución del Colegio de Bachilleres de Chiapas, COBACh, fundado en 1997. La situación educativa en las 
comunidades y parajes es distinta, en ellas sólo se encuentran el nivel preescolar y primarias bilingües, muchas de ellas incompletas, también escuelas multigrados fundadas a partir de la década de 1970, en un proceso continuo que no ha cesado. Otra modalidad educativa que atiende la educación inicial, preescolar y primaria, a partir de 1994, es la que imparte el Consejo Nacional de Fomento Educativo, con su modalidad de «educación comunitaria».

Cada modalidad educativa responde a una política determinada desde el Estado, en mi tesis sólo me ocupé de analizar la educación primaria. Una de las escuelas de la cabecera municipal, «Benito Juárez», ${ }^{7}$ fue fundada a partir de la década de 1930, como parte de las denominadas Misiones culturales o Escuelas rurales, que a Chiapas llegaron más tarde si se compara con otros estados de la República; este tipo de escuelas surgidas de los ideales revolucionarios tenían como finalidad la integración de la «familia mexicana» y el objetivo específico de «atacar de manera integral el problema de la rehabilitación económica y la organización social de nuestra población campesina», según las palabras del presidente Calles (citado por Aguirre 1992: 89). Este es el inicio de los ensayos estatales del integracionismo.

La modalidad de las escuelas bilingües-biculturales de forma más clara forman parte de las políticas integracionistas que tienen como antecedente inmediato los Centros Coordinadores Indigenistas del Instituto Nacional Indigenista, y en Chiapas llegan en 1951. Es a partir de los trabajos de INI en materia educativa cuando, en la segunda mitad de la década de 1970, se funda lo que ahora se conoce como Dirección General de Educación Indígena, DGEI, institución que retoma el sistema de enseñanza bilingüe-bicultural con el que inició INI su labor desculturizadora ampliamente cuestionada en su tiempo.

Finalmente, Conafe es la modalidad educativa que, en particular en las regiones Altos y Selva de Chiapas, comienza a operar a partir del levantamiento armado del EZLN —en otras regiones del país, esta modalidad ya operaba desde la década de 1970_, en 1994, y que llega, al igual que en otros estados del país, operando con base en los «programas compensatorios» en materia educativa, proyecto que se inscribe como uno más en las 
políticas de Estado. En muchos sentidos Conafe, al igual que después el Programa de Educador Comunitario Indígena, PECI, fueron políticas estatales contrainsurgentes, o «para taparle el ojo al macho» en la región de conflicto, en materia educativa, dado que no se hicieron efectivos por parte del gobierno federal los Acuerdos de San Andrés firmados en febrero de 1996.

\section{EDUCACIÓN ESCOLAR Y CAMBIO DE VALORES CULTURALES}

La educación escolar en las culturas indígenas ha tenido fuertes consecuencias para la vida comunitaria, principalmente por el cambio de valores que ha introducido. El trabajo realizado en San Andrés Sakamch'en, Larráinzar, buscó tratar de explicar el proceso de resignificación de las identidades de género, etnia y clase a partir de la educación escolar. Una ejemplificación práctica de estos cambios de valores fue el trabajo realizado con los niños y niñas de sexto grado en la escuela Benito Juárez de la cabecera municipal, donde con base en la aplicación de una encuesta a 38 niñas y 39 niños — que eran los/as alumnas que conformaban el grado en los dos grupos existentes-, que tenía como objetivo conocer la forma en que niños y niñas perciben su realidad inmediata y las formas en que valoran, a partir de su identidad sexual, las relaciones de género, las de clase y etnia - los resultados pueden ser revisados en la tesis ya mencionada-; es decir, cómo se perciben como niños o niñas — sobre la base de las actividades que creen que son «naturales» de acuerdo con el sexo con el que se identifican; si se asumen o no como indígenas, y cómo perciben las relaciones entre los/as indígenas y los/as mestizos/as —o ladinos/as- Debido a razones de espacio y por considerar de mayor relevancia para los objetivos de este trabajo, sólo señalaré que el material me permitió analizar cómo los/as niños/as van aprendiendo y aprehendiendo que lo que les inculcan sus maestros y maestras es el único conocimiento válido - la cultura occidental-, y que lo que ellos/as poseen como cultura carece de valor. Los/as niños/as ante preguntas específicas relacionadas con la lengua en que prefieren que les enseñen y el tipo de maestro/a que prefieren que les enseñe, etc., muestran que prefieren el español y al profesor mestizo, más de $90 \%$ de los/as niños/as responde a favor de esto. 
Por su parte, las posturas que asumen los/as maestros/as tienen que ver con el conocimiento y/o desconocimiento teórico y práctico que presentan de la cultura del pueblo donde prestan sus servicios, que se traducen en declaraciones contradictorias acerca de las prácticas culturales de la población del lugar donde trabajan:

...algunas costumbres no son tan buenas, porque son medio agresivas, a no ser que nosotros no sepamos el significado, eso sí que cada quien, lo que podemos ver para nosotros puede ser agresivo, pero qué tal que para ellos no, ¿verdad? Una de ellas es cuando lo del carnaval, matan el gallo, pues, con los dientes, pasan y lo muerden hasta que le quitan la cabeza, o sea, para nosotros no lo vemos bien, pero para ellos, tal vez porque nosotros no sepamos el significado, entonces digo, o tal vez lo que faltaría es que nosotros los maestros lo viéramos, ya consiste en cada una de sus tradiciones y costumbres que ellos tienen, pero ya que lo sepamos bien, ya podemos opinar, esto sirve, esto no sirve, digo y (entrevista con una profesora de una escuela primaria general o monolingüe, 2001).

Respecto a la situación del uso de la lengua indígena y el español, los/as maestros/as también tienen posturas contradictorias, al igual que los padres y madres de familia que se inclinan porque la enseñanza de sus hijos e hijas sea en español, hay quienes se postulan porque la enseñanza de los niños/as sea en su lengua familiar-comunitaria. Las posturas, sean de un tipo o de otro, todas coinciden en que la lengua indígena tendría un uso limitado al ámbito familiar comunitario y que en consecuencia el fin último de la educación, el que los niños y niñas tienen que aprender, es el uso del español para poder insertarse en las relaciones con ámbitos más amplios. Esta misma concepción sigue prevaleciendo en la educación intercultural que se está practicando en Chiapas; es decir, los/as profesores/as consideran que las culturas indígenas no tendrán posibilidad de participar de la diversidad cultural y la identidad nacional — «la unidad en la diversidad»— al mismo tiempo, si no son capaces de castellanizarse.

\section{LOS ACUERDOS DE SAN ANDRÉS Y LA EDUCACIÓN AUTÓNOMA}

La lucha actual de los pueblos indígenas por la libre determinación y por el reconocimiento de los derechos y las culturas indígenas tiene que inscribirse como temática a considerar dentro de la interculturalidad. Así lo demandaron los, las zapatistas para Chiapas y para el resto de los pueblos indígenas de México y el mundo, demandas que quedaron signadas en 
los Acuerdos de San Andrés. El proyecto de educación intercultural debe plantearse a partir de que las culturas indígenas de México tengan la libertad de reconstruir sus culturas, que se encuentra deterioradas o trastocadas después de cinco siglos de imposiciones, de segregación y olvidos. Para la práctica de la interculturalidad se requiere que las culturas sean autónomas, primero se tiene que lograr que en las culturas indígenas de México no existan sujeción a la cultura hegemónica, eso es un derecho que ha sido negado por la «nobleza de Estado», que a partir del monopolio de la «res pública»-República- a nombre de todos/as los/as mexicanos/as creen saber lo que conviene a los pueblos indígenas. ¿Cómo pueden en la interculturalidad las culturas relacionarse, intercambiar elementos de su cultura, si la definición misma de cultura se impone como parte de una política de Estado?

Para desarrollar parte de lo que constituye la educación en autonomía es importante señalar cuál es la definición de autonomía propuesta en los Acuerdos de San Andrés de 1996:

La autonomía es la expresión concreta del ejercicio del derecho a la libre determinación expresada como un marco que se conforma como parte del Estado Nacional. Los pueblos indígenas podrán en consecuencia decidir su forma de gobierno interna y sus maneras de organizarse política, social, económica y culturalmente. Dentro del nuevo marco constitucional de autonomía se respetará el ejercicio de la libre determinación de los pueblos indígenas en cada uno de los ámbitos y niveles en que la hagan valer, pudiendo abarcar uno o más pueblos indígenas, conforme a las circunstancias particulares y específicas de cada entidad federativa. El ejercicio de la autonomía de los pueblos indígenas contribuye a la unidad y democratización de la vida nacional y fortalecerá la soberanía del País (Inciso II de las «Propuestas Conjuntas que el Gobierno Federal y el EZLN se comprometen a enviar a las instancias de debate y decisión nacional, correspondiente al punto 1.4 de las Reglas de Procedimiento»).

En los Acuerdos de San Andrés la educación ocupa un lugar importante como parte de los derechos y cultura indígena, y existe un compromiso del gobierno federal en materia educativa que me parece importante citar, porque es dentro de este marco que los zapatistas comenzaron a construir su proyecto educativo; aquí se propone: 
Asegurar educación y capacitación. El Estado debe asegurar a los indígenas una educación que respete y aproveche sus saberes, tradiciones y formas de organización. Con procesos de educación integral en las comunidades que les amplíen su acceso a la cultura, la ciencia y la tecnología; educación profesional que mejore sus perspectivas de desarrollo; capacitación y asistencia técnica que mejore los procesos productivos y calidad de sus bienes; y capacitación para la organización que eleve la capacidad de gestión de las comunidades. El Estado deberá respetar el quehacer educativo de los pueblos indígenas dentro de su propio espacio cultural. La educación que imparta el Estado debe ser intercultural. Se impulsará la integración de redes educativas regionales que ofrezcan a las comunidades la posibilidad de acceder a los distintos niveles de educación ( $5^{\circ}$ punto de «Los compromisos del Gobierno Federal con los Pueblos Indígenas». Acuerdos de San Andrés, 1996).

En el $4^{\circ}$ punto de las Propuestas conjuntas que el Gobierno Federal y el EZLN se comprometieron a enviar a las instancias de debate y decisión nacional, correspondiente al apartado 1.4 de las reglas de procedimiento, se hace referencia a la necesidad de una educación integral indígena, en el que existe el compromiso estatal de «respetar el quehacer educativo» de los pueblos indígenas y de asignar «recursos financieros, materiales y humanos», además de que las comunidades y pueblos indígenas tendrán el derecho de «seleccionar, ratificar y remover a sus docentes», basándose en criterios académicos y de desempeño profesional, bajo acuerdos entre las autoridades educativas y los pueblos y comunidades. Se entiende, entonces, que debe construirse un nuevo modelo educativo dentro del marco autónomo, además:

Se ratifica el derecho a la educación bilingüe intercultural de los pueblos indígenas. Se establece como potestad de las entidades federativas, en consulta con los pueblos indígenas, la definición y desarrollo de sus programas educativos con contenidos regionales, en los que deben reconocer su herencia cultural. Por medio de la acción educativa será posible asegurar el uso y desarrollo de las lenguas indígenas, así como la participación de los pueblos y comunidades de conformidad con el espíritu del Convenio 169 de la OIT ( $4^{\circ}$ punto de las Propuestas conjuntas que el Gobierno Federal y el EZLN se comprometieron a enviar a las instancias de debate y decisión nacional, correspondientes al apartado 1.4 de las reglas de procedimiento. Acuerdos de San Andrés, 1996).

Existió también un acuerdo tripartito de compromisos para Chiapas entre el gobierno del estado de Chiapas, el gobierno federal y el EZLN, correspondiente al punto 1.3 de las 
reglas de procedimiento, en el que se hace referencia a la necesidad de fomentar una «Educación indígena bilingüe intercultural», y donde se señala que:

El reconocimiento de la composición pluricultural del estado de Chiapas, así como del derecho de los pueblos indígenas a que se respeten, promuevan y difundan los elementos significativos que constituyen su cultura, plantea la necesidad de que en la Constitución Local se incorpore, por un lado, el derecho de los chiapanecos a recibir una educación conforme a la letra y espíritu del artículo $3^{\circ}$ Constitucional federal y su reglamentaria de la Ley General de Educación; por otro lado, en el marco de las reformas que se plantean a la Constitución Federal, es necesario que la reforma local considere lo siguiente:

a) Que la educación que reciban todos los chiapanecos, difunda y promueva la historia, costumbres, tradiciones, y todos aquellos valores componentes de nuestra raíz cultural e identidad nacional.

b) La educación indígena debe ser bilingüe e intercultural.

c) El estado debe asegurar a los indígenas una educación que respete y aproveche sus saberes, tradiciones y formas de organización.

d) Respetar el quehacer educativo de los pueblos indígenas dentro de su espacio cultural.

e) Que en la organización y formulación de los planes y programas de estudio tengan participación prioritaria los pueblos indígenas, en lo que se refiere a contenidos regionales y sobre diversidad cultural. Estas modificaciones implican la reforma del artículo $4^{\circ}$ de la Constitución de Chiapas («Compromisos para Chiapas del Gobierno del estado y federal y el EZLN, correspondientes al apartado 1.3 de las reglas de procedimiento». Acuerdos de San Andrés, 1996).

Finalmente, en los Acuerdos de San Andrés existen compromisos y propuestas conjuntas de los gobiernos estatal, federal y el EZLN, en los que se señalan las acciones y medidas para Chiapas. En este último punto, en materia de educación, se apunta la importancia de la creación de instituciones indígenas que se hagan cargo de la difusión, divulgación y desarrollo de las lenguas indígenas, y que «traduzcan obras científicas, técnicas y culturales», además se señala la importancia de modificar los materiales educativos para las comunidades y pueblos indígenas dado que los actuales libros de texto no reflejan la realidad de los pueblos y culturas indígenas. Bajo estos fundamentos y debido a que el gobierno federal incumplió los Acuerdos, los zapatistas iniciaron la construcción del proyecto educativo autónomo. 
La educación es pues dentro del marco de la autonomía una práctica cultural resignificada, sujeta a cambios, no obstante a partir del análisis de lo que se ha conseguido con la construcción de la Escuela Rebelde Autónoma Zapatista, ESRAZ, $1^{\circ}$ de Enero, en la comunidad Oventik, del municipio de San Andrés, analizada por su significación social, dentro del campo educativo de este municipio, en consecuencia la educación autónoma significa, pues, todo un reto para las comunidades indígenas que tienen que plantearse cuestionamientos profundos como: ¿Para qué queremos educarnos? ¿Qué queremos aprender y para qué nos va a servir? ¿Quiénes nos van a enseñar? Sus fundamentos ideológicos son:

\begin{abstract}
Nosotros no queremos seguir el camino de la educación oficial, porque en vez de ser un lugar de transmisión de conocimiento se convierte en un lugar para olvidar nuestro conocimiento. El maestro oficial quiere que los niños dejen de hablar su lengua familiar-comunitaria y que la comunidad se avergüence de ser indígena desconociendo a sus pasados, padres y familiares, quiere que olvidemos lo que nuestros abuelos nos han enseñado con tanta paciencia y dedicación en los primeros años de vida (Proyecto Semillita de Sol, s/f).
\end{abstract}

Queremos una educación que no sea clasista, sexista y racista, en la cual nos tomen como sujetos y no como objetos. La educación debe construir al hombre y a la mujer nuevos, sin explotación, marginación, opresión o desprecio, y reconociendo todos los derechos que merecemos como pueblos. Debe ser una educación en donde se hable de la situación real que vivimos, y no debe ser alienante, explotadora o abusiva en contra de nuestras culturas (Fichas de trabajo de la Escuela Secundaria Rebelde Zapatista $1^{\circ}$ de Enero).

El inicio de la práctica educativa en autonomía para los zapatistas significó, en un contexto más amplio, la des-obligación estatal de satisfacer un derecho fundamental, como lo es la educación. Esta des-obligación se tradujo en una situación cómoda para el Estado que no se vio en la necesidad de pagar a los/as maestros/as, de prepararlos/as, de construir la infraestructura básica, de proporcionar los materiales para la práctica educativa.

Por otra parte, la educación autónoma ha construido un escenario favorable para la resignificación de las identidades de género, etnia y clase, como un proceso dialéctico de cuestionamiento a las imposiciones de la cultura propia y de la ajena. Así podemos 
entender cómo se dio la construcción de las «identidades rebeldes» de las y los indígenas zapatistas que cuestionaron al Estado por condenar a los pueblos indígenas a vivir en estado permanente de subordinación, y a la cultura propia por haber contribuido a perpetuar esa subordinación. El análisis de su situación llevó a casi la mitad de los/as habitantes del pueblo de San Andrés a sumarse a la causa zapatista. Este análisis de las condiciones reales que existen en la práctica del sistema de cargos, cuestionando su función objetiva basada en la asignación de cargos tradicionales a las personas que han estudiado poco o a las que carecen de estudios, mientras que aquellas personas que han adoptado otra ideología religiosa y aquellos que tienen un grado de estudio más elevado no se les nomina en el sistema de cargos, especialmente los cargos que presuponen fuertes gastos económicos y actividades que implican mayor dedicación en tiempo y esfuerzos.

Las condiciones reales de la diferenciación social, que dan paso a las rupturas, conflictos y divisionismos familiares y comunitarios, fueron el caldo de cultivo, sumado a las causas externas del sistema capitalista y las nuevas formas de relacionarse que introducen las instituciones como los partidos políticos y los distintos grupos religiosos que fungen como articuladoras de las comunidades indígenas con la sociedad en general.

\section{RESULTADOS. TRES FORMAS DE EDUCAR EN UN SOLO ESPACIO}

Los significados de la educación escolar se han impuesto a través de un largo proceso de inculcación-introyección como un valor en sí mismo, como un instrumento de superación personal, sin embargo, los procesos diferenciados de las culturas —relatividad culturalnos enseñan, al analizar los procesos de resignificación de las identidades, de género, etnia y clase, que en las culturas indígenas los cambios en las significaciones y en los paradigmas existenciales que se han impuesto en las relaciones sociales — y que se han naturalizado-; son cambios de percepción que actúan con otro ritmo.

La educación escolar en un contexto específico, como el municipio de San Andrés Sakamch'en, nos muestra que ésta se impone como cultura de clase sobre los valores culturales locales. Así se van trasformando los escenarios, las percepciones de la realidad, existen cambios en las relaciones familiares y se opaca - cuando no se suprime- la 
enseñanza del modelo educativo indígena, de la enseñanza de la tradición oral — de los/as ancianos/as se pasa al conocimiento sistematizado en los libros-; la escuela ha introducido «...un cambio de lenguajes, una modificación del sentido común. No más la tierra y la casa, no más la voz sino la escritura y un lugar de aprendizaje separado de aquel en que se vive han reconstruido las jerarquías cognoscitivas ... del saber indígena al saber nacional» (Gorza 1998: 33); de la enseñanza teórica y práctica del trabajo para la vida se pasa al conocimiento abstracto del aula del profesor o profesora. Todo esto también dio paso a pensar en una nueva forma de educación: la educación para la autonomía.

\section{LAS IDENTIDADES EN EL MARCO DE LA PRÁCTICA DE LA INTERCULTURALIDAD}

Hasta hoy, la educación escolar en las regiones indígenas no ha permitido a los alumnos/as pensar reflexivamente sobre sus realidades — más bien ha contribuido a encapsularlos en la identidad de indígenas de una manera colonizada-. Se necesita un proceso de desnaturalización de lo socialmente construido para que, a partir de la identidad indígena, se pueda pensar reflexivamente sobre las posibilidades de optar por otras identidades, como deseo y no como imposición; como señala atinadamente Tzvetan Todorov, las personas «puede[n] ir de un lugar a otro, puede[n] adaptarse a culturas distintas. Cuando miramos a los mestizos o los híbridos se les mira un poco con desprecio, sin embargo, no hay un solo ser humano que no sea un lugar de encuentro de varias culturas» (2001: 5).

\section{COMO INDÍGENAS ¿PARA QUÉ QUEREMOS FORMARNOS? SUMISIÓN, ETNOCIDIO ¿NUEVOS} ESCLAVOS PARA EL SIGLO XXI?

La escuela se ha convertido en el referente de la modernidad para las comunidades indígenas, y ni siquiera las posturas más radicales — como es el caso de los y las integrantes del EZLN - ponen en duda la escuela y la educación como un valor social, como una «conquista social» que se convierte en bandera de las luchas sociales contra el Estado.

Existen fundamentos antropológicos de la educación que conciben al hombre y a la mujer como seres educables — que enseñan a la vez que aprenden (Freire 1999: 22), como seres en proceso de formación, «la educación juega un papel de medio de comunicación de la cultura a nivel social» (Janer y Colom 1995: 78). La educación estimula la adquisición de 
los elementos culturales como un proceso mediante los cuales un grupo humano trasmite a las jóvenes generaciones el bagaje cultural que representa su herencia cultural (ibíd.); o de la forma en la que nos lo ha mostrado Bourdieu, la educación es el proceso relacional de «comunicación pedagógica, o sea, de la imposición y de la inculcación de una arbitrariedad cultural según un modelo arbitrario de imposición y de inculcación —educación— (1996: 46). Como proceso relacional, la educación contribuye a la reproducción de la sociedad y en consecuencia de las culturas.

Desde este punto de vista, la educación está cargada de mitos y de atribuciones, por ello las exigencias sociales que esperan y exigen la solución de problemas prácticos como los problemas de clase, de género, etnia y generacionales; en pocas palabras, la mitificación de la educación contribuye a su propia «perdición». Por otra parte, concebir la educación como proceso relacional de reproducción social y cultural nos permite analizar la perpetuación de las relaciones desiguales de clase, género y etnia. Cuando los/as integrantes de una cultura trasmiten su herencia, incluyen en ésta una visión particular del mundo, unas categorías particulares de división y clasificación del mundo social, de las relaciones de etnia, clase y género, especialmente las categorías que se refieren a las formas masculina y femenina de clasificar el mundo y de reproducirlo; así se educan a los hijos bajo los conceptos del mundo masculino y a las hijas se les inculcan las categorías del mundo femenino, todo ello va acompañado de una perpetuación de las relaciones desiguales e inequitativas entre hombres y mujeres y se asignan funciones sociales diferenciadas. Por ejemplo, las mujeres son las encargadas, por mandato social, trascendente, de preservar las tradiciones, y por ello de vivir en desigualdad social con los hombres, y en un ámbito más amplio a existir como clase oprimida.

Para el Estado mexicano, históricamente, la existencia de los pueblos indígenas ha constituido un estorbo para las políticas modernizadoras, por ello se ha propuesto la educación como el instrumento de aculturación, lo cual en la práctica se ha traducido en etnocidio cultural. Al otorgar educación de mala calidad a la población, en general, se preparan las condiciones para que exista la servidumbre que requiere el sistema capitalista neoliberal que, como modelo económico, se impone cual paradigma existencial y en su 
afán de universalización, lo que menos importa es respetar las identidades culturales - no solamente las identidades indígenas, sino también la población negra, y las poblaciones marginadas y segregadas, en general一, ¿en qué situación quedan aquí las culturas indígenas?

\section{LOS PRINCIPALES RETOS DE UNA POLÍTICA INTERCULTURAL PARA EL FORTALECIMIENTO ÉTNICO CON IGUALDAD Y EQUIDAD DE CLASE, ETNIA Y GÉNERO}

Existe un proceso de evolución multiforme de las culturas y también de las identidades culturales. Como señala Todorov (2001), la supresión de las identidades individuales no es posible ni deseable, pues la supresión significaría deshistorizar a las personas y a los grupos. Lo deseable, en todo caso, es el fortalecimiento de las identidades culturales, como un proceso que vaya acompañado de la fórmula razón más identidad o identidad con razón (Díaz-Polanco 2001); es decir, que el asumir y defender una identidad no se convierta en un encapsulamiento para las personas o grupos, sino que la opción por una identidad, y a partir de ahí de las identidades que se deseen asumir, sea un proceso reflexivo que los potencien y fortalezcan como personas, culturas y sociedades.

Para construir el modelo de educación —intercultural o con el complemento que le quieran poner- se tiene que tomar como punto de partida el análisis y la búsqueda del mejoramiento de la situación actual de las culturas indígenas. En el caso de Chiapas, éstas se encuentran enmarcadas en un contexto de desigualdades sociales, de género y generacionales, de intolerancia y discriminación por las preferencias sexuales, de un conflicto no resuelto sino esquivado por el Estado - la reivindicación zapatista), etc., que constituyen contextos reales donde se imparte la educación, en una sociedad con agentes en posiciones desiguales que no han aprendido a convivir - porque la mayoría apenas sobrevive- - En la interculturalidad será necesario dar cabida a la diversidad cultural y tomar en cuenta que las culturas no existen independientemente de las relaciones sociales que las crean y de las que a la vez son creadoras.

Históricamente, las relaciones entre los pueblos indígenas de México, con el resto de la sociedad mexicana y con el Estado mexicano, se han caracterizado por la desigualdad, 
mientras no se planteen mecanismos que modifiquen las condiciones de desigualdad social, y se pugne por la equidad en las relaciones que tienen como fundamento una ideología discriminatoria — basada en sistemas simbólicos binarios: mestizos-patrones/indígenassirvientes; mestizos-ricos/indígenas-pobres; mestizos-cultos/indígenas-ignorantes, que adquieren el grado de prescripciones trascendentes- y una base económica desigual, que se justifica como diferencia cultural - los indios por ser indios son pobres, son pobres porque son haraganes, etc.-, imaginarios y prácticas que naturalizan lo social y que impiden que, por ahora, existan las condiciones necesarias para la práctica efectiva de la interculturalidad.

Por otra parte, no somos ajenos/as a las contradicciones en las que se enmarca cualquier formación social. La interculturalidad requiere del hecho de que las culturas tengan cierto grado de autonomía, hecho que en nuestro país se ha negado a las culturas indígenas, por decisión estatal; así se siguen imponiendo desde el Estado los programas encaminados a sacar de la marginación, la pobreza endémica, a las poblaciones indígenas a través de proyectos políticos construidos desde las instituciones estatales. ¿Pero, son los programas desarrollistas: educativos, económicos y culturales del Estado lo que la población indígena requiere? ¿Se han tomado en cuenta los problemas sociales y los proyectos que como culturas se formulan los pueblos indígenas? Lo que existe en materia educativa son interrogantes que habría que analizar y responder y sobre las que hay que trabajar conjuntamente. ¿Cómo unificar criterios ante la existencia de modalidades educativas -educación bilingüe-bicultural -o intercultural-, educación monolingüe -en castellano- y Conafe -educación «comunitaria»-, Programa de Educador Comunitario Indígena, PECI-, cuyos objetivos, en muchos casos son contradictorios?, y de competencia entre sí. Las contradicciones entre modalidades educativas se traducen en competencias por ampliar su oferta educativa; se constituyen también en elementos en disputa entre las comunidades: por la pertenencia étnica de los maestros y maestras, por las ideologías de las que son portadores/as los maestros/as mestizos/as y los/as maestros/as indígenas; en fin, por los intereses que cada modalidad defiende ante la comunidad, etc. 
Todas estas diferencias cualitativas se traducen en incomprensiones, en estigmatizaciones, en conflictos y competencias que imposibilitan un diálogo entre todos los agentes que participan de la educación escolar. ¿Cómo construir los fundamentos de la interculturalidad cuando los modelos prevalecientes, los significados de la educación, son referentes de las imposiciones culturales que ahora se pretenden borrar; si los subsistemas escolares -que responden a una etapa histórica concreta - se han esclerotizado y convertido en monopolios de las burocracias educativas y de sus agentes?

Para el Estado, la interculturalidad es todo un reto, es dar paso a una democracia práctica, no discursiva; como señala Gimeno (2001: 18) al Estado no le corresponde decidir qué valores orientarán a la sociedad $-\mathrm{y}$ a las culturas-, sino garantizar las condiciones para que los grupos — sociales y pueblos indios- hagan efectivos sus proyectos.

Para los/as educadores/as, la puesta en práctica de la educación intercultural significa también un reto que consiste en resignificar la práctica de la docencia, asumirla como un proceso dialéctico de aprender al enseñar y enseñar al aprender (Freire 1999). Hasta ahora la práctica de la docencia, para muchos/as maestros/as, ha sido una obligación, una carga que ha sido pesada porque la sociedad capitalista ha creado necesidades, no vocaciones, y desde el punto de vista de una obligación ha habido coerción, imposiciones.

Para los/as integrantes de las diferentes culturas que conformando la nación mexicana la enriquecen, la práctica de la interculturalidad significa también un doble reto. Por una parte está la necesidad de trabajar sobre un proceso de resignificación y reconstrucción de la cultura, y por otro tomar enseñanzas de nuestra historia pasada. Considerar que la trasmisión de los elementos culturales y de la visión indígena del mundo es un proceso en constante cambio, que lo que nos enseñaron nuestros padres y madres fue una verdad válida para la época en que ellos crecieron y vivieron, pero que las realidades actuales exigen la adecuación de nuestras culturas a los procesos sociales actuales, sólo de esta forma comenzaremos a vivir nuestra identidad cultural no como una carga, que en muchos casos significa fanatizar nuestras culturas, la defensa de las «costumbres» no debe significar manipulación de la identidad cultural. 
La educación intercultural es también todo un reto para el campo científico, el no existir como un «mundo aparte» del común, sino a partir de los análisis de realidades concretas realizar aportes, no imponer «verdades» a los procesos sociales. La interculturalidad deberá considerar la interdisciplinaridad como una necesidad y no encerrarse en la visión puramente cultural, porque las culturas no existen en un vacío, sino sustentadas en relaciones sociales concretas. Actualmente se abren nuevas perspectivas de análisis con los estudios sobre trasculturalidad (Hopenhaym 2000), y «entreculturalidad» (Corona Berkin 2007) en la educación, con los que los debates, en hora buena, se están enriqueciendo. 


\section{BIBLIOGRAFÍA}

Aguirre Beltrán, Gonzalo, 1992, Teoría y práctica de la educación indígena, Universidad Veracruzana/Instituto Nacional Indigenista/Gobierno del estado de Veracruz/Fondo de Cultura Económica, México (Obra antropológica X).

Bourdieu, Pierre y Jean-Claude Passeron, 1996, La Reproducción. Elementos para una teoría del sistema de enseñanza, $2^{\mathrm{a}}$ edición, Ed. Fontamara, México.

2000, La Dominación Masculina, Traducción de Joaquín Jordá, Ed., Anagrama, Barcelona.

Coordinación General del Plan Puebla Panamá, 2001, Plan Puebla Panamá. Documento Base, Capítulo México, Coordinación General del Plan Puebla Panamá.

Corona Berkin, Sarah y otras voces, 2007, Entre voces... Fragmentos de educación «entrecultural», Universidad de Guadalajara, México.

Díaz Polanco, Héctor, 2001, «Discordia en la familia liberal. La identidad y la razón», en Diálogos Latinoamericanos, n. 004, Universidad de Aarhus, Latinoamericanistas, pp. 2136.

Enlace Civil, A. C., s/f, «Proyecto Semillita de Sol», Enlace Civil, A. C., San Cristóbal de Las Casas, Chiapas, en http://www.enlacecivil.org.mx/pr_e_semillita.html [consulta: abril 2002].

Escuela Secundaria Rebelde Autónoma Zapatista, E.S.R.A.Z., 2001, Oventik Aguascalientes II. Nuestra Lengua, Nuestra Memoria. Ideas para clases de Tsotsil, Edición Escuela Secundaria Rebelde Autónoma Zapatista, San Andrés Sakamch'en, Chiapas, México.

s/f, «Fichas de trabajo de la Escuela Secundaria Rebelde Zapatista (ESRAZ) $1^{\circ}$ de Enero», Documento inédito. 
Freire, Paulo, 1999, La educación como práctica de la libertad, 48 edición, Siglo XXI Editores, México.

Gimeno Sacristán, José, 2001, «Políticas y prácticas culturales en las escuelas: los abismos de la etapa posmoderna», Revista Fundamentos en humanidades, año II, v. 2, n. 4, Universidad Nacional de San Luis, Argentina, pp. 7-43.

Gómez Lara, Horacio, 2002, «Educación, identidad y género en San Andrés Sakamch'en (Larráinzar), Chiapas», Tesis de licenciatura, Facultad de Ciencias Sociales, Universidad Autónoma de Chiapas, San Cristóbal de Las Casas, Chiapas.

Gorza, Piero, 1998, «El dibujo, el bolígrafo y la tierra», Anuario de Estudios Indígenas VII, IEI-UNACH, San Cristóbal de Las Casas, pp. 25-38.

Hopenhayn, Martín, 2000, «Transculturalidad y Diferencia. -El lugar preciso es un lugar movedizo-》, Revista Cinta de Moebio, n. 7, Facultad de Ciencias Sociales, Universidad de Chile, Chile, en http://rehue.csociales.uchile.cl/publicaciones/moebio/07/frames05.htm [consulta: enero de 2002].

Janer, G. y A. J. Colom, 1995, «El modelo cultural en la construcción de la Antropología de la Educación», en Cuestiones de Antropología de la Educación, coordinado por Noguera, J., Ceac, Barcelona, pp. 63-94.

Pérez Pérez, Elías, 2000, «La Crisis de la Educación Indígena en el área Tzotzil en Los Altos de Chiapas». Tesis de maestría, San Cristóbal de Las Casas, Chiapas.

Presidencia de la República, s/f, Acuerdos de San Andrés, «Derechos y Cultura Indígena», Presidencia de la República, México, en http://zedillo.presidencia.gob.mx/ pages/chiapas/docs/sanandres.html [consulta: diciembre de 2001]. 
Todorov, Tzvetan, 2001, «Destinos de la Identidad», Letras Libres, España, en http://www.letraslibres.com/index.php?art=7072 [consulta: febrero de 2002].

Tomasevski, Katarina, 2002, «Mercantilizar la educación, tendencia del gobierno foxista», La Jornada, lunes 29 de julio, México.

\section{Notas}

${ }^{1}$ La nominación de «San Andrés Sakamch'en de los Pobres» es la que aplican los y las simpatizantes y/o militantes zapatistas a su espacio social [municipio autónomo]; mientras que la nominación San Andrés Larráinzar la utiliza la población no zapatista.

${ }^{2}$ De entrada, la distinción de educación bilingüe intercultural, pensada sólo para los pueblos indígenas significa la persistencia de las desigualdades culturales y sociales que subyacen a la práctica educativa en México.

${ }^{3}$ Sylvia Schmelkes fundó y tuvo a su cargo, desde sus inicios, en el año 2001 y hasta el año 2007, la Coordinación General de Educación Intercultural Bilingüe, CGEIB, de la Secretaría de Educación Pública, SEP. La ponencia a que hago alusión fue presentada en el marco del Foro «Los Retos de la Educación Intercultural en México», celebrado en San Cristóbal de Las Casas, el 3 de julio de 2001, meses antes de que se creara CGEIB.

${ }^{4}$ El PPP desde 2004 pasó a denominarse «Proyecto de Integración y de Desarrollo de Mesoamérica».

${ }^{5}$ En una entrevista para el periódico La Jornada, Tomasevski, señaló: «El gobierno mexicano ha incluido el presupuesto privado dentro de sus metas para llegar a 8 por ciento del PIB ... Desde el punto de vista de los derechos humanos esto es un desastre, porque se mezclan el gasto privado, la compra y venta de la educación, con la inversión pública, que es responsabilidad del gobierno. De esa forma la cifra de inversión es buena, pero no es consecuencia de que toda la gente tenga su derecho a la educación, sino de que los ricos pagan por su educación. No se vale incluir el presupuesto privado, porque el derecho a la educación debe ser regulado como una responsabilidad pública» (La Jornada, 29 de julio de 2002). Además, alerta acerca de que convertir la educación en una mercancía no contribuye a eliminar las diferencias socioeconómicas, sino que las acrecienta, pues lleva a la existencia de «un sistema educativo doble: educación buena para aquellos que pueden pagar y mala para los pobres» (ibíd.).

${ }^{6}$ Estos principios son procesos paralelos y acompañan a las y los indígenas a lo largo de sus vidas. La metodología para la reconstrucción del proceso de ETI que aquí utilizo se basa en la tesis de maestría del profesor indígena tsotsil, originario del municipio de Chenalhó, Elías Pérez (2000).

${ }^{7}$ La otra escuela donde se realizó el trabajo es «Fray Matías de Córdoba y Ordóñez», que también funciona en la cabecera municipal, la cual comenzó a funcionar desde principios de 1980 como anexo de la escuela «Benito Juárez», y en 1986 obtuvo su registro como escuela independiente.

Fecha de recepción: 10 de agosto de 2010.

Fecha de aceptación: 6 de diciembre de 2010. 